J.Pharm.Dyn., 5, 727-733 (1982)

\title{
MOTOR DYSFUNCTION IN SPONTANEOUS THYMOMA RATS, BUFFALO/MNA
}

\author{
FUMINORI KATO AND MinORU WATANABE \\ Department of Chemical Pharmacology Faculty of Pharmaceutical Sciences, Nagoya City University, \\ Tanabe-street, Mizuho-ku, Nagoya 467, Japan
}

(Received May 10, 1982)

The muscle responsiveness of rats of Buffalo/Mna strain with spontaneous thymoma (Mna rats) was investigated in vivo electrophysiologically and pharmacologically in order to determine the principal cause of the muscle weakness and fatigability. Hindlimb muscles of these rats exhibited a lesser contractility and a greater fatigability during repetitive nerve stimulation than those of rats of another strain, ACI. Nevertheless, there was no difference in the time course of amplitude of evoked electromyograms between Mna and ACI rats. According to the test of drug sensitivity, Mna rats were much less sensitive to curare than ACI rats. Sensitivity to 4-aminopyridine was normal. In conclusion, Mna rats showed an increased fatigability and weakness of the muscle. The principal cause seems to be involved in contractile mechanisms subsequent to muscle cell membrane. The reduced sensitivity to curare suggests a possibility of some change at the site of neuro-muscular junction.

Keywords - Buffalo/Mna rats, thymoma, muscle fatigability, muscle weakness, $d$ tubocurarine subsensitivity

\section{INTRODUCTION}

Recent electronmicroscopic investigations have revealed the presence of cells resembling striated muscle cells in human thymus. ${ }^{1,2)}$ Therefore, much attention has recently been paid to the relationship between the thymus and muscle diseases. Matsuyama et al. have established a rat strain of high incidence of thymoma and named it Buffalo/Mna strain. ${ }^{3)}$ The thymoma began to develop from 36 weeks after birth, and the incidence was $100 \%$ in 18 months old male rats, and histologically the thymoma closely resembled human thymomas.) They have observed that almost all young rats of this strain show a slight abnormality of walking on the hind legs and also that a majority of adult male rats of this strain show a remarkably lesser ability to remain on the revolving roller of a rotarod apparatus than rats of other strains. ${ }^{3,5)}$ Ultrastructural examination of the extraocular muscle revealed an elongation and ramification of the postsynaptic membranes. ${ }^{5,6}$ Also in the femoral muscles, the following changes have been observed: muscle fibers vary- ing in size; distention of the sarcoplasmic reticulum; formation of lipid droplets; and detachment and distortion of myofibrils and myofilaments. ${ }^{7)}$ These findings are very interesting in connection with muscle and nerve diseases.

In the present study, several electrophysiological and pharmacological investigations of the hindlimb muscles of the thymoma rats were performed in order to determine the principal site of the defect resulting in an increased muscle fatigability, and the results were discussed about which of the neuron, neuro-muscular junction and muscle progressively degrades in these rats.

\section{MATERIALS AND METHODS}

Experiment 1 (Exp. 1) and Experiment 2 (Exp. 2) were performed without and with surgical invasion, respectively, using rats of two strains in a room kept at $25^{\circ} \mathrm{C}$.

Exp. 1 Isotonic Measurements of Muscle Fatigability and Electromyographical Evaluation - Ten male rats of Mna strain (350-450 g body weight, $41-47$ weeks of age) were used in this study. 
Eight male rats of ACI strain $(300-360 \mathrm{~g}$ body weight, 51-82 weeks of age) served as control. Animals were anaesthetized by intraperitoneal injection of urethane $(0.75 \mathrm{~g} / \mathrm{kg})$, alpha-chloralose $(20 \mathrm{mg} / \mathrm{kg}$ ) and thiopental $(25 \mathrm{mg} / \mathrm{kg})$, and were mounted on an apparatus.

(1) Measurement of Muscle Movement and Fatigability: Repetitive electrical stimulation was carried out by an electronic stimulator (NihonKoden, MSE-20S) using stainless steel needle electrodes implanted percutaneously near the lateral popliteal nerve (peroneal nerve) of the right leg. Supramaximal stimuli $(0.2 \mathrm{~ms}$ in duration) were applied at $2 \mathrm{~Hz}$ for $20 \mathrm{~min}$. Electrical stimulation elicited dorsiflexion (movement as kicking up), which was inhibited completely by a dose of $0.05 \mathrm{mg} / \mathrm{kg}$ of $d$-tubocurarine chloride $\left(d-\mathrm{Tc}_{c}\right), i . v$, and was recovered rapidly and completely by a dose of $0.2 \mathrm{mg} / \mathrm{kg}$ of edrophonium, $i$.v. The muscle contractile movement was measured by means of a hand-made isotonic transducer, and recorded on a pen recorder (Nihon-Koden, WI-180). The transducer consisted of a small piece of magnet on the axis of an isotonic lever and a magnetic-electric converting element (hole IC). The linearity was enough for the present experiment. The load was nearly null. Muscle fatigability during repetitive stimulation was evaluated by comparing response at each scheduled time (10 and $20 \mathrm{~min}$ ) with the maximal response attained soon after the initiation of the stimulation.

(2) Electromyographical Evaluation: Electromyographic study was performed approximately $30 \mathrm{~min}$ after the measurement of muscle fatigability. The right leg was fixed to a fixing bar using an adhesive tape, and steel needles were inserted as recording electrodes into the belly and the tendon of the anterior tibialis muscle. Supramaximal stimuli $(0.2 \mathrm{~ms}$ in duration) were applied on the peroneal nerve at $10 \mathrm{~Hz}$ for $90 \mathrm{~s}$, and subsequently at $0.2 \mathrm{~Hz}$ for $4 \mathrm{~min}$. Compound muscle action potentials were displayed on an oscilloscope via a DC amplifier (San-Ei Instrument Co., model 6L), and were recorded on a film. The peak to peak amplitude of the evoked potential was measured. Fluctuation of the amplitude of EMG during repetitive stimulation was used for the evaluation of fatigability, i.e. the response at each scheduled time $(5,20$, and $40 \mathrm{~s}$, and $1,1.5,2,2.5,3.5$, and $5.5 \mathrm{~min}$ ) were compared with a single response before the repetitive stimulation.

Exp. 2 Isometrical Measurement of Muscle Fatigability, Electromyographical Evaluation and Drug Sensitivity — Seven male rats of Mna strain (280-430 g body weight, 40-54 weeks of age) and four male rats of ACI strain (250-350 g body weight, $31-93$ weeks of age) were used in this study.

(1) Measurement of Muscle Contractile Force and Electromyogram and Evaluation of Fatigability: Animals were anaesthetized by urethane $(1.0 \mathrm{~g} / \mathrm{kg}$, i.p. $)$ and alpha-chloralose $(12.5 \mathrm{mg} / \mathrm{kg}$ ), and were fixed on their back. Respiration was assisted by means of an endotracheal cannula and an artificial respirator (Takashima Ltd.) throughout the experiment. The left sciatic nerve was cut and the distal end was stimulated through a bipolar platinum electrode. The tendon of the anterior tibialis muscle was freed from the left leg and was connected to a force displacement transducer via a cotton thread, and the twitch tension was recorded on a pen recorder. Electromyograms were simultaneously recorded using $\mathrm{Ag}-\mathrm{AgCl}$ electrodes placed on the belly and the tendon of the anterior tibialis muscle. Supramaximal stimuli of $0.2 \mathrm{~ms}$ in duration were applied at $2 \mathrm{~Hz}$ for 10 min. For the evaluation of muscle fatigability, twitch tension and the amplitude of EMG at 5 and $10 \mathrm{~min}$ were compared with the maximal contractile response attained during the course of the stimulations and a single electrical response before repetitive stimulation, respectively.

(2) Sensitivity to Drugs: Drugs were dissolved in $0.9 \% \mathrm{NaCl}$ solution and injected into the right femoral vein. $d-\mathrm{Tc}, 0.05 \mathrm{mg} / \mathrm{kg}$, was given intravenously $20 \mathrm{~min}$ after the measurement of muscle fatigability, and the sensitivity to $d$-Tc was evaluated by comparing the maximally reduced tension with the preinjectional response. 
4-Aminopyridine (4-AP), $1 \mathrm{mg} / \mathrm{kg}$, was given intravenously 20 or $30 \mathrm{~min}$ after the injection of $d$-Tc, and sensitivity to 4-AP was evaluated by comparing twitch tensions at 5 and 20 min with the preinjectional response.

Statistics - Values were expressed as mean \pm s.e.m. and statistical significance was tested with Aspin-Welch method, a modified Student's $t$-test.

Drugs - The following drugs were used: urethane, alpha-chloralose, $d$-tubocurarine chloride ( $d-\mathrm{Tc}$ ) and 4-aminopyridine (4-AP) (all from Wako Pure Chemicals), thiopental sodium (Tanabe), and edrophonium chloride (Kyorin).

\section{RESULTS}

Isotonic Measurement of Muscle Fatigability and Electromyographical Evaluation (Exp. 1)

(1) Measurement of Muscle Fatigability - Time course of the muscle contractile response (shortening) during repetitive stimulation (at $2 \mathrm{~Hz}$ for $20 \mathrm{~min}$ ) of the lateral popliteal nerve is illustrated in Fig. 1. Contractile response increased gradually to a small extent during a few minutes after the start of repetitive stimulation, and slowly declined thereafter. Maximal response and responses at 10 and $20 \mathrm{~min}$ were used for subsequent evaluations and the results are shown in Fig. 2A. All these responses in Mna rats were smaller than the corresponding responses in ACI rats. The differences between these pairs of

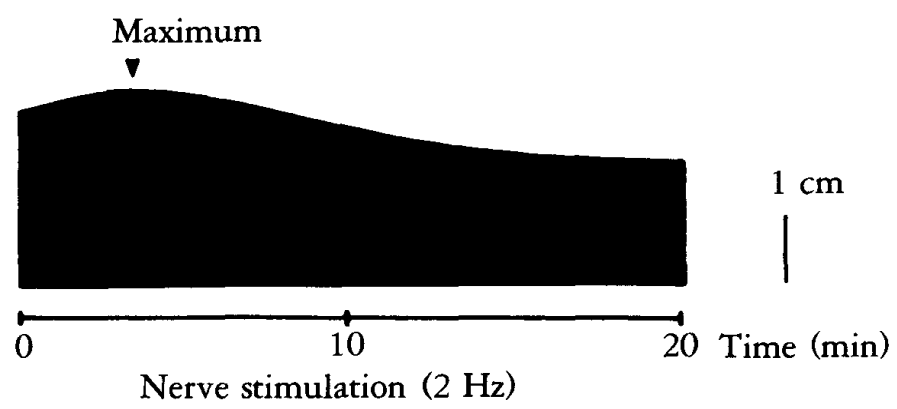

FIG. 1. Time Course of Muscle Contractile Responses during Repetitive Stimulation of the Lateral Popliteal Nerve (at $2 \mathrm{~Hz}$ for $20 \mathrm{~min}$ ) responses were highly significant $(p<0.01$ or 0.001 ). Decrease in the contractile movement of the muscle (muscle fatigability) of rats of each strain, expressed in terms of per cent of the max-
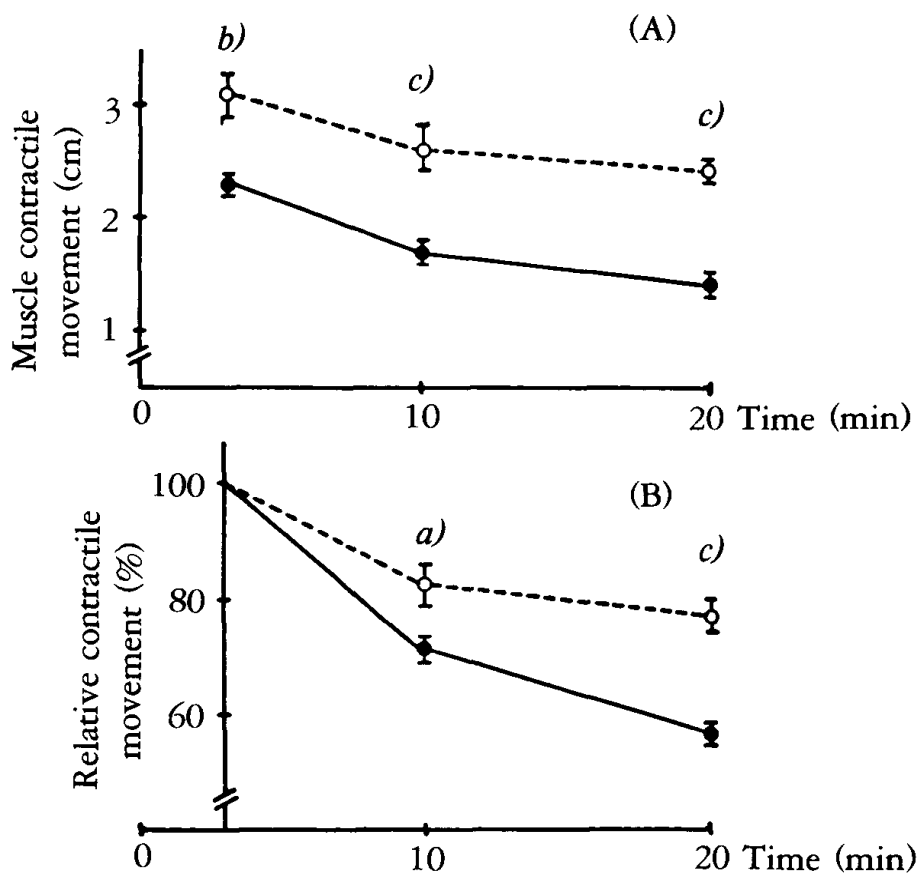

FIG. 2. Comparison of Contractile Decrements during Repetitive Stimulation of the Lateral Popliteal Nerve on Mna (O) and ACI Rats (O)

Each point with bar represents the mean \pm S.E.; $a), b)$, and c) indicate statistical difference from control at $p<0.05,0.01,0.001$, respectively.

(A) Absolute evaluation of muscle contractile movement.

(B) Relative evaluation of muscle contractile movement in terms of per cent maximal response (muscle fatigability).

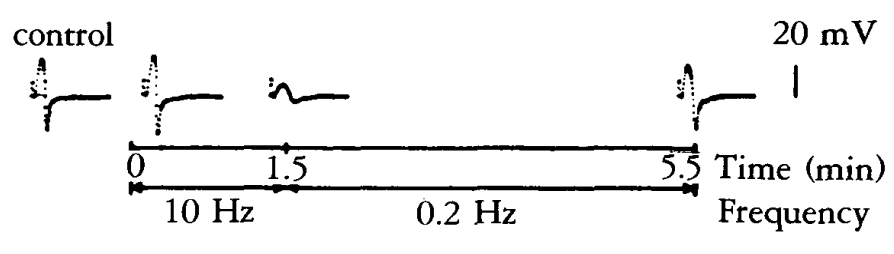

FIG. 3. Time Course of the Amplitude of EMG during Repetitive Stimulation of the Lateral Popliteal Nerve (at $10 \mathrm{~Hz}$ for $1.5 \mathrm{~min}$ and at $0.2 \mathrm{~Hz}$ for 4 min) 
imal response, is shown in Fig. 2B. Mna rats showed a severer fatigability of the muscle than ACI rats $(p<0.001)$.

(2) Electromyographical Evaluation - Time course of the amplitude of EMG during repetitive stimulation (at $10 \mathrm{~Hz}$ for $1.5 \mathrm{~min}$, and subsequently at $0.2 \mathrm{~Hz}$ for $4 \mathrm{~min}$ ) is illustrated in Fig. 3 .

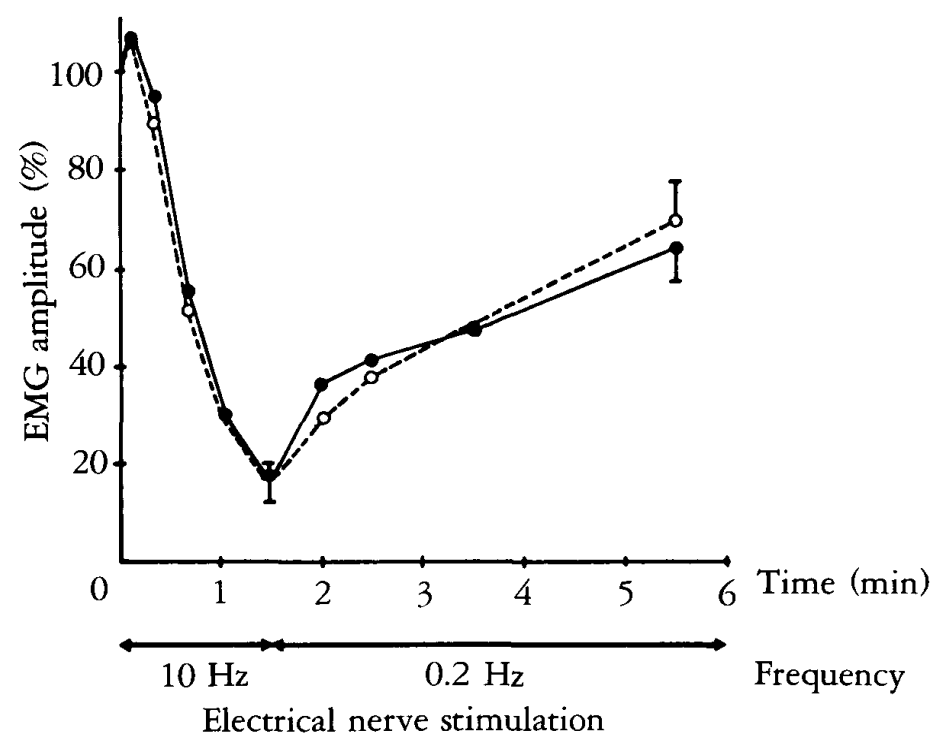

FIG. 4. Comparison of the Time Course of the Amplitude of EMG during Repetitive Stimulation of the lateral Popliteal Nerve between Mna (O) and ACI Rats (O)

Amplitude of single response before repetitive stimulation was regared as 100.

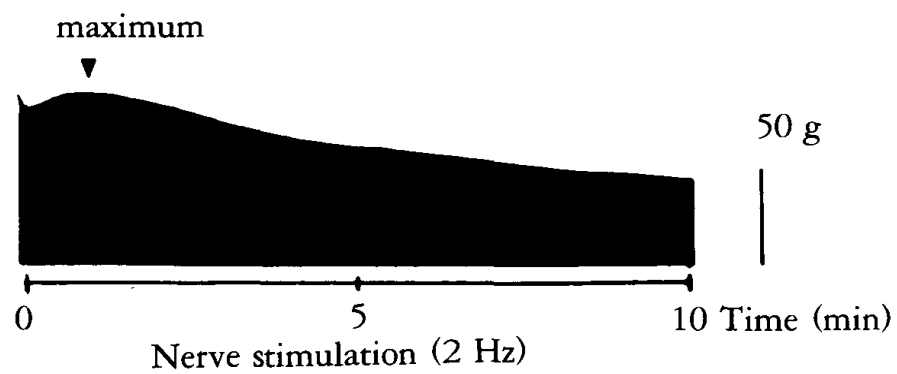

FIG. 5. Time Course of Twitch Tension of the Anterior Tibialis m. during Repetitive Stimulation of the Sciatic Nerve (at $2 \mathrm{~Hz}$ for $10 \mathrm{~min}$ )
Simultaneously with the start of the stimulation, the amplitude of EMG increased slightly, reached a maximum within $10 \mathrm{~s}$, and then rapidly declined. When the frequency of stimulation was lowered from $10 \mathrm{~Hz}$ to $0.2 \mathrm{~Hz}$, the amplitude of EMG was gradually recovered toward control level. The amplitude of EMG at each scheduled time was expressed in terms of \% of that of single response before the start of repetitive stimulation . The result in Mna rats, during the periods of both attenuation and recovery, was almost identical with that in ACI rats (Fig. 4). The amplitude of control response in Mna rats $(44.1 \pm 4.6 \mathrm{mV})$ was significantly smaller $(p<0.01)$ than that in ACI rats. $(55.4 \pm 4.1 \mathrm{mV})$.

Isometrical Measurement of Muscle Fatigability and Electromyographical Evaluation (Exp. 2)

(1) Measurement of Muscle Fatigability - Time course of twitch tension of the anterior tibialis muscle during repetitive stimulation (at 2 $\mathrm{Hz}$ for $10 \mathrm{~min}$ ) of the sciatic nerve, is illustrated in Fig. 5. Twitch tension began to increase moderately following an initial transient decline, reached maximum around a minute, and then gradually and appreciablly declined. The amplitude of twitch tension at the peak and those at 5 and $10 \mathrm{~min}$ are shown in Fig. 6A. The amplitude at $10 \mathrm{~min}$ in Mna rats was significantly lower $(p<0.01)$ than that in ACI rats. When twitch tension per $100 \mathrm{~g}$ body weight of the two strains was compared, as shown in Fig. 6B, the value in Mna rats was significantly less than that in ACI rats throughout the experiment. When the degree of decline in amplitude of contraction was assessed, there was no significant difference in the value of relative twitch tension at $5 \mathrm{~min}$ between the two strains, but the value at $10 \mathrm{~min}$ in Mna rats was significantly smaller $(p<0.05)$ than that in ACI rats (Fig. $6 \mathrm{C}$ ), again suggesting that the fatigability is exaggerated in Mna rats.

(2) Electromyographical Evaluation - EMG of the anterior tibialis muscle was recorded simultaneously with tension. The amplitude of single response before repetitive stimulation (control response) and those of responses at 5 and 10 min were measured for subsequent evaluation. 


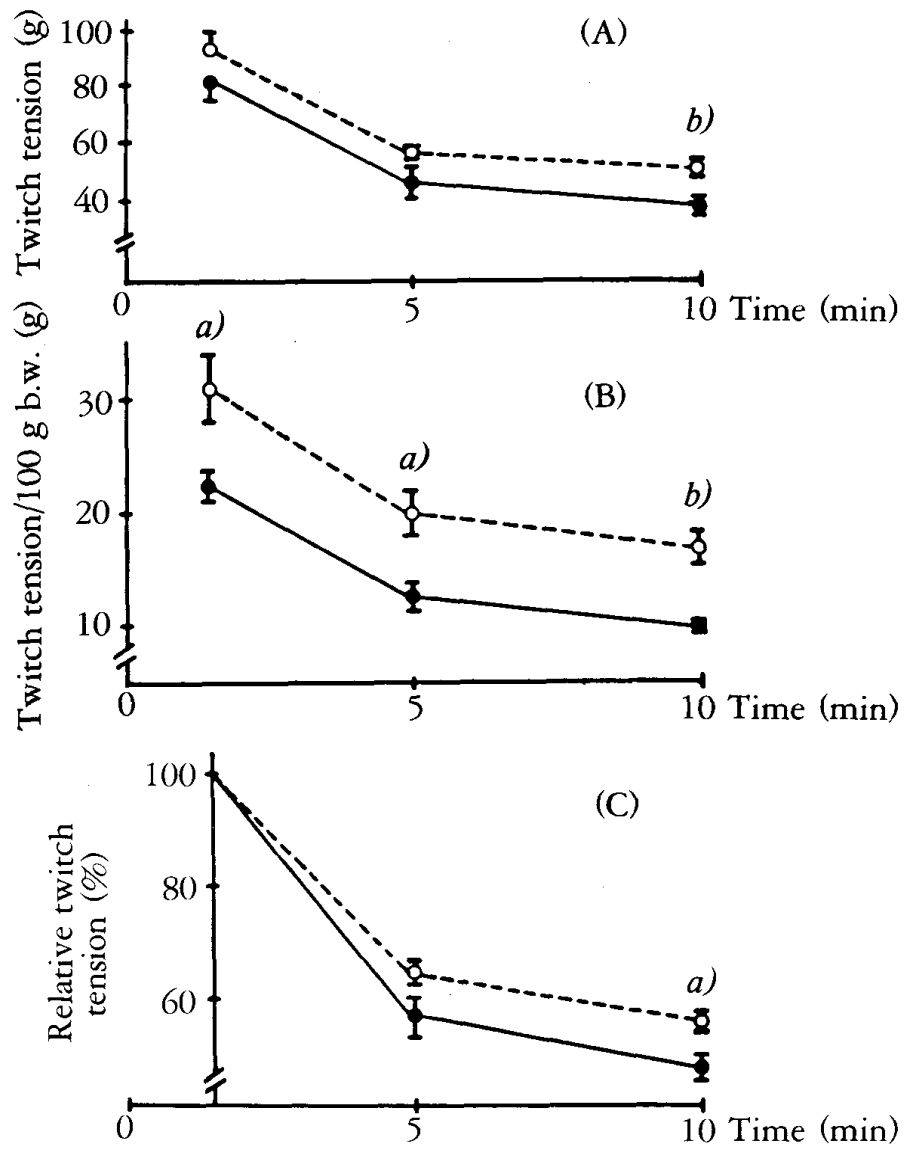

FIG. 6. Comparison of Contractile Decrements during Repetitive Stimulation of the Sciatic Nerve on Mna (O) and ACI Rats (O)

(A) Absolute evaluation of twitch tension.

(B) Absolute evaluation of twitch tension per 100 $g$ body weight.

(C) Relative evaluation of twitch tension (maximal tension $=100$ ).

a) and b) indicate significant difference from control at $p<0.05,0.01$, respectively.

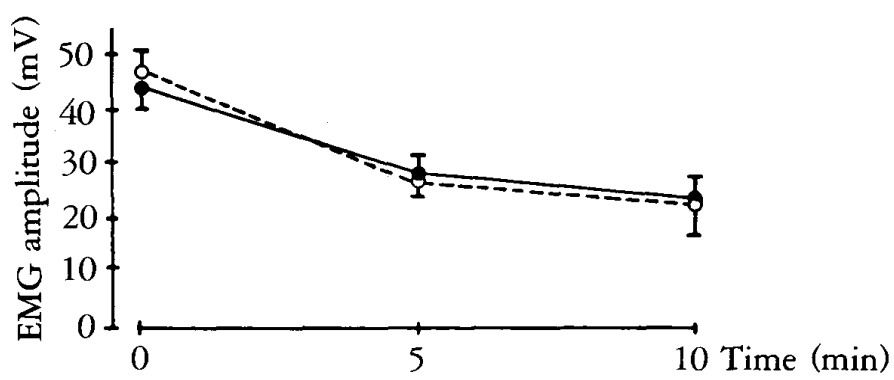

FIG. 7. Comparison of the Amplitude of EMG during Repetitive Stimulation of the Sciatic Nerve on Mna (O) and ACI Rats (O)
The results are shown in Fig. 7. There was no significant difference between the two strains; nor was there when the amplitude of EMG was expressed in terms of per cent of the amplitude before repetitive stimulation. In the initial period of the repetitive stimulation, myasthenia gravis like-decremental response of EMG was not observed in rats of each strain.

(3) Sensitivity to Drugs — First, sensitivity of rats of each strain to intravenous $d$-Tc was examined. In Mna rats the minimum tension after the injection was $56 \pm 4 \%$ of the tension before the injection, but the value in control rats was $9 \pm 2 \%$, indicating that the sensitivity of Mna rats was highly significantly lower $(p<0.001)$ than that of ACI rats.

Second, sensitivity of rats to 4-AP was examined. In Mna rats the twitch tensions 5 and 20 min after the injection were $118.5 \pm 4.3 \%$ and $146.6 \pm 5.4 \%$ of the tension before the injection, respectively, and the values in control rats were $118.0 \pm 2.3 \%$ and $148.0 \pm 3.1 \%$, respectively. This agent enhanced the twitch tension to an almost equal extent in both strains.

\section{DISCUSSION}

In both Exp. 1 and 2, Mna rats showed a severer fatigability of muscle than ACI rats which served as controls (Fig. 2B and 6C). Furthermore, the results in Fig. $2 \mathrm{~A}$ and $6 \mathrm{~B}$ indicate that Mna rats possess a lesser muscle contractility, and a lesser relative contractile force of muscle per body weight than ACI rats. These results appear to explain the finding of Matsuyama et al. that Mna rats showed a lesser ability to remain on the revolving roller of a rotarod apparatus than rats of other strains. ${ }^{3,5)}$ Possible site for abnormality or etiology of such muscular disease characterized by these symptoms are: (1) neurogenic, (2) presynaptic, (3) postsynaptic, (4) myogenic abnormalities.

As shown in Fig. 4, the decremental response and recovery in EMG amplitude during repetitive stimulation observed in Mna rats were similar to those in ACI rats. When twitch tension and EMG were measured simultaneously, Mna rats showed a severer decrement of contractile 
response than ACI rats, nevertheless both rats of strains were similar in the time course of EMG amplitude (Fig. 7). No myasthenic decremental response of EMG in the initial period of the repetitive stimulation was observed in these rats. Since EMG reflects the compound action potential response which is generated on the muscle cell membrane, the finding that EMG elicited by nerve stimulation is normal indicating generally that the processes of nerve, neuro-muscular junction and muscle cell membrane are normal. Therefore, these findings suggest that the principal cause of muscle fatigability is involved in the sequence of events subsequent to those occurring on the muscle cell membrane. This suggestion is in agreement with the fact that various submicroscopical changes have been demonstrated in the extraocular muscles ${ }^{5,6)}$ and the femoral muscles $^{7)}$ : degenerated muscle cells, elongation of the sarcoplasmic reticulum, appearance of giant mitochondria, lipid droplets, myelin bodies, and amorphous substance between myofibrils, and detachment and distortion of myofibrils. These changes also suggest some submembranous site of dysfunction.

As observed in the examination of sensitivity to $d$-Tc, however, Mna rats showed a lower sensitivity than ACI rats. An elongation and ramification of the postsynaptic membrane of the motor endplates have also been observed submicroscopically in the extraocular muscles. ${ }^{5,6)}$ Therefore, a possibility remains that Mna rats have some postsynaptic change in leg muscles which could not be detected by electromyographical method. The change, if any, should be different from those in myasthenia gravis (MG), since observed were neither increased sensitivity to $d$-Tc nor the myasthenic decremental response of evoked EMGs which are commonly observed in MG patients. ${ }^{89}$ ) In addition, the sensitivity of Mna rats to 4-AP was similar to that of ACI rats. This result failed to indicate that the transmission was blocked either pre- or post-synaptically, because 4-AP has been suggested to potentiate transmitter release from the motor nerve terminal mainly by increasing the quantum content, ${ }^{10,11}$ ) and effectively counteract or reverse postsynaptic as well as presynaptic blockade of transmission. ${ }^{12,13)}$

There are several kinds of useful animal models for the investigation of muscle and nerve diseases. Rats of experimental autoimmune myasthenia gravis (EAMG) ${ }^{14,15)}$ and dystrophic mice, ${ }^{16)}$ for instance, have helped much to advance the etiologies of MG and muscular dystrophy, respectively. These animals can never be ultimate models of many types of the diseases. On the other hand, there are some interesting findings on the relationship between thymic abnormality and muscle diseases. The one is that human $\mathrm{MG}$ is often complicated with thymoma, ${ }^{17,18)}$ and recent electronmicroscopic investigations have revealed the presence of cells resembling striated muscle cells in the human thymus. ${ }^{1,2)}$ Another is that murine dystrophy is complicated with thymic abnormality. ${ }^{19,20}$ ) The relationship between them, however, remains unresolved. From these points of view, Mna rats which spontaneously develop thymoma and also a fatigability and a weakness of muscle should give a clue for the elucidation of the muscle diseases.

In conclusion, Mna rats with thymoma showed an exacerbated fatigability and weakness of muscle, and the principal cause seems to be involved in contractile mechanisms subsequent to the events in muscle cell membrane. The reduced sensitivity to curare suggests a possible functional change at the site of NMJ undetectable by electromyographic studies.

Acknowledgments

The authors wish to express their thanks to Dr. M. Matsuyama, Aichi Cancer Center Research Institute, for valuable advice as well as for supplying Buffalo/Mna and ACI rats used in this work.

\section{REFERENCES}

1) K.Henry: Mucin secretion and striated muscle in the human thymus, Lancet, i, 183-185 (1966).

2) Thea Feltkamp-Vroom: Myoid cells in human thymus, Lancet, i, 1320-1321 (1966).

3) M.Matsuyama and T.Nagayo: Development of 
thymoma and myasthenia in Buffalo/Mna rats, Proc. Japan. Cancer Assoc., 36, 30 (1977).

4) S.Yamada, K.Masuko, M.Ito and T.Nagayo: Spontaneous thymoma in Buffalo rats, Gann, 64, 287-291 (1973).

5) M.Matsuyama, H.Ito, Y.Masuyama, T.Nakamura and M.lida: Spontaneous muscle weakness in high thymoma Buffalo/Mna rats, Nagoya Med. J., 23, 93-98 (1978).

6) Y.Masuyama: Ultrastructural changes in the extraocular muscles of thymoma-prone Buffalo/Mna strain rats, Acta Soc. Ophthalmol. Jap., 82, 843-953 (1978).

7) M.Matsuyama, H.Amo, C.Yamada, F.Kato and M.Watanabe: Myopathy in rats of the high thymoma Buffalo/Mna strain, Research Committee of Experimental Models for Intractable Diseases, 85-91 (1979).

8) F.F.Foldes, D.H.Klonymus, W.Maisel and K.E.Osserman: A new curare test for the diagnosis of myasthenia gravis, $J A M A, 203,133-137$ (1968).

9) S.H.Horowitz, G.Genkins, P.Kornfeld and A.E. Papatestas: Regional curare test in evaluation of ocular myasthenia, Arch. Neurol., 32, 84-88 (1975).

10) P.Illes and S.Thesleff: 4-Aminopyridine and evoked transmitter release from motor nerve endings, $\mathrm{Br} . \mathrm{J}$. Pharmac., 64, 623-629 (1978).

11) H.Lundh: Effects of 4-aminopyridine on neuromuscular transmission, Brain Res., 153, 307-318 (1978).

12) H.Lundh, S.Leander and S.Thesleff: Antagonism of the paralysis produced by botulinum toxin in the rat. The effect of tetraethylammonium, guanidine and 4aminopyridine, J. Neurol. Sic., 32, 29-43 (1977).

13) Y.I.Kim, M.M.Goldner and D.B.Sanders: Facilitatory effects of 4-aminopyridine on normal neuromuscular transmission, Muscle and Nerve, 3, 105-111 (1980).

14) J.M.Lindstrom, B.L.Einarson, V.A.Lennon and M.E.Seybold: Pathological mechanisms in experimental autoimmune myasthenia gravis. 1. Immunogenicity of syngeneic muscle acetylcholine receptor and quantitative extraction of receptor and antibody-receptor complexes from muscles of rats with experimental autoimmune myasthenia gravis, J. Exp. Med., 144, 726-738 (1976).

15) J.M.Lindstrom, A.G.Engel, M.E.Seybold, V.A.Lennon and E.H.Lambert: Pathological mechanisms in experimental autoimmune myasthenia gravis. 2. Passive transfer of experimental autoimmune myasthenia gravis in rats with anti-acetylcholine receptor antibodies, J. Exp. Med., 144, 739-753 (1976).

16) A.M.Mechelson, E.S.Russel and P.J.Harman: Dystrophia muscularis: a hereditary primary myopathy in the house mouse, Proc. Natl. Acad. Sci. U.S., 41, 1079-1084 (1955).

17) J.A.Simpson: An evaluation of thymectomy in myasthenia gravis, Brain, 81, 112-144 (1958).

18) J.A.Simpson: Myasthenia gravis: a new hypothesis, Scot. Med. J., 5, 419-436 (1960).

19) T.A.Kretser and B.G.Livett: Evidence of a thymic abnormality in murine muscular dystrophy, Nature (London), 263, 682-684 (1976).

20) D.F.Horrobin, R.O.Morgan, R.A.Karmali, A.I.Ally, M.S.Manku, M.Karmazyn and S.C.Cunnane: Thymic changes in muscular dystrophy and evidence for an abnormality related to prostaglandin synthesis or action, Ann. N. Y. Acad. Sci., 317, 534-549 (1979). 